\title{
Surpassing the standard quantum limit for optical imaging using nonclassical multimode light
}

Treps, N.; Andersen, Ulrik Lund; Buchler, B.; Lam, P.K.; Maitre, A.; Bachor, H.A.; Fabre, C.

Published in:

Physical Review Letters

Link to article, DOI:

10.1103/PhysRevLett.88.203601

Publication date:

2002

Document Version

Publisher's PDF, also known as Version of record

Link back to DTU Orbit

Citation (APA):

Treps, N., Andersen, U. L., Buchler, B., Lam, P. K., Maitre, A., Bachor, H. A., \& Fabre, C. (2002). Surpassing the standard quantum limit for optical imaging using nonclassical multimode light. Physical Review Letters, 88(20), 203601. https://doi.org/10.1103/PhysRevLett.88.203601

\section{General rights}

Copyright and moral rights for the publications made accessible in the public portal are retained by the authors and/or other copyright owners and it is a condition of accessing publications that users recognise and abide by the legal requirements associated with these rights.

- Users may download and print one copy of any publication from the public portal for the purpose of private study or research.

- You may not further distribute the material or use it for any profit-making activity or commercial gain

- You may freely distribute the URL identifying the publication in the public portal 


\title{
Surpassing the Standard Quantum Limit for Optical Imaging Using Nonclassical Multimode Light
}

\author{
N. Treps, ${ }^{1,2}$ U. Andersen, ${ }^{2,3}$ B. Buchler, ${ }^{2}$ P. K. Lam, ${ }^{2}$ A. Maître, ${ }^{1}$ H.-A. Bachor, ${ }^{2}$ and C. Fabre ${ }^{1}$ \\ ${ }^{1}$ Laboratoire Kastler Brossel, Université Pierre et Marie Curie, case 74, 75252 Paris cedex 05, France \\ ${ }^{2}$ Department of Physics, Faculty of Science, Australian National University, Canberra ACT 0200, Australia \\ ${ }^{3}$ Department of Physics, Technical University of Denmark, DK-2800 Kongens Lyngby, Denmark
}

(Received 18 January 2002; published 3 May 2002)

\begin{abstract}
Using continuous wave superposition of spatial modes, we demonstrate experimentally displacement measurement of a light beam below the standard quantum limit. Multimode squeezed light is obtained by mixing a vacuum squeezed beam and a coherent beam that are spatially orthogonal. Although the resultant beam is not squeezed, it is shown to have strong internal spatial correlations. We show that the position of such a light beam can be measured using a split detector with an increased precision compared to a classical beam. This method can be used to improve the sensitivity of small displacement measurements.
\end{abstract}

DOI: 10.1103/PhysRevLett.88.203601

It has long been known that optical measurements are ultimately limited in their sensitivity by quantum noise, or shot noise, of the light. For more than a decade the usage of nonclassical light has provided ways of improving the sensitivity beyond this standard quantum limit [1]. For example, squeezed light has been used to improve interferometric [2] and absorption [3] measurements. However, these improvements can be applied only to signals that correspond to the time modulation of light, as they rely on the temporal quality of the light. On the other hand, many applications require spatial measurement of light. While improvements for spatial applications based on nonclassical light have been proposed theoretically [4,5], no experimental demonstration has yet been shown to work with continuous wave light. The challenge is to create strong spatial correlations within a laser beam, rather than the temporal correlation typically found in nonclassical light sources [6]. While some experiments involving sub-Poissonian vertical cavity surface admitting lasers operating in a transverse multimode regime exhibited a nonrandom spatial distribution of the quantum noise [7], no spatial correlation was observed within the produced beam. Here we present the first successful experimental demonstration of a spatially ordered light source and a measurement of the spatial modulation of a laser beam position to below the standard quantum limit in the continuous wave regime.

This experimental work builds on theoretical work done on nonclassical multimode states of light [8]. Such states display strong spatial correlations, and their productions have been the subject of extensive studies in recent years [9]. In particular, the process of parametric down conversion in a nonlinear optical medium has been extensively studied, as it produces "twin photons" which are quantum correlated both temporally and spatially. Such strong spatial quantum correlations in the plane perpendicular to the direction of propagation are produced in spontaneous down conversion [10] and in multimode transverse optical parametric oscillators [11]. Nevertheless, to our knowledge,
PACS numbers: 42.50.Dv, 42.30.-d, 42.50.Lc

there has been no experimental demonstration of quantum correlations with a multimode transverse light in the continuous wave regime.

Precision optical imaging using CCD cameras or photodetector arrays is required in many areas of science, ranging from astronomy to biology. Ultimately, the performance of optical imaging technology is limited by quantum mechanical effects. Of particular importance, as far as applications are concerned, is the measurement of image displacements, for example, the position of a laser beam. Techniques that rely on determining the position of a laser spot include atomic force microscopy [12], measurement of very small absorption coefficients via the mirage effect [13], and observation of the motion of single molecules [14]. These measurements are usually performed as shown in Fig. 1. The beam is incident on a split detector that delivers two currents proportional to the light intensity integrated over the two halves $(x<0$ and $x>0)$ of the image plane. If the beam is initially centered on the detector, the mean value of the photocurrent difference is directly proportional to the relative displacement $d$ of the beam with respect to the detector. With a classical, shot

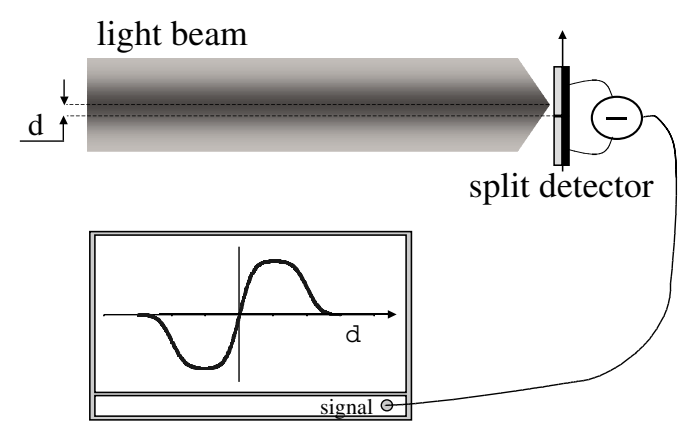

FIG. 1. Measurement of the displacement of a light beam. A split detector measures the intensities of the two halves of the image plane. When the beam is nearly centered, the difference between these intensities gives a signal that is proportional to the beam displacement $d$. 
noise limited laser source, the smallest displacement that can be measured (with a signal-to-noise ratio of one) is shown to be [4]

$$
d_{\mathrm{SQL}}=\frac{\sqrt{N}}{2 I(0)} .
$$

Here $N$ is the total number of photons recorded by the two detectors during the measurement time, and $I(0)$ is the local density of photons (photons per unit transverse length) at the position of the boundary between the two detectors. For a $\mathrm{TEM}_{00}$ Gaussian beam with radius $w_{0}$, the minimum measurable displacement is found to be

$$
d_{\mathrm{SQL}}=\sqrt{\frac{\pi}{8}} \frac{w_{0}}{\sqrt{N}} .
$$

For maximum focusing of the Gaussian beam, $w_{0}=\lambda$, and we obtain $d_{\mathrm{SQL}} \approx \lambda / \sqrt{N}$, which is the absolute minimum displacement of a physical system that can be measured with classical beams [12]. Equation (2) shows that a more powerful laser, or a longer measurement time, gives increased measurement precision. However, in many applications these alternatives are simply not practical. In the case of atomic force microscopy, for example, excessive laser power ultimately leads to radiation pressure noise [15]. For biological applications, large laser power may damage the samples under investigation and an increased integration time leads to loss of bandwidth. This is the motivation for looking for alternative methods of increasing measurement precision.

The limit of Eq. (2) can be surpassed only using multimode nonclassical light. Let us consider a beam of light with an electric field distribution given by $E(x)$. We can build an orthonormal basis of the transverse plane $\left\{u_{i}\right\}$ such that $u_{0}=E(x) /\|E(x)\|$ is the first vector; $u_{1}$ is a "flipped" mode, given by $-u_{0}(x)$ for $x<0$ and $u_{0}(x)$ for $x>0$ (see Fig. 2); and the other modes are chosen in order to form a basis. In this basis, the mean field of our light lies only in the first mode $u_{0}$ but, a priori, all of the modes contribute to the quantum noise. In order to determine the relevant modes of our measurement, we consider the interference quantities between two modes on each half of the split detector:

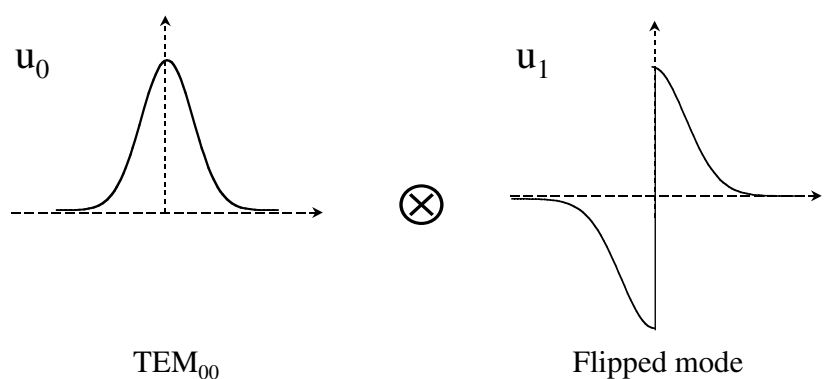

FIG. 2. Electric field profile of the two constituent modes used to form the nonclassical multimode beam.

$$
\begin{aligned}
& I_{x<0}\left(u_{i}, u_{j}\right)=\int_{-\infty}^{0} u_{i}^{*}(x) u_{j}(x) d x, \\
& I_{x>0}\left(u_{i}, u_{j}\right)=\int_{0}^{+\infty} u_{i}^{*}(x) u_{j}(x) d x .
\end{aligned}
$$

Then the interference quantities relevant for a total measurement (sum of the two photodetectors) and a differential measurement (difference of the two photodetectors) can be written as follows:

$$
\begin{aligned}
& I_{\text {sum }}\left(u_{i} u_{j}\right)=I_{x<0}\left(u_{i}, u_{j}\right)+I_{x>0}\left(u_{i}, u_{j}\right), \\
& I_{\text {diff }}\left(u_{i} u_{j}\right)=I_{x<0}\left(u_{i}, u_{j}\right)-I_{x>0}\left(u_{i}, u_{j}\right) .
\end{aligned}
$$

One can then show that for any transverse mode $u_{i}$,

$$
I_{\text {sum }}\left(u_{i} u_{1}\right)=I_{\mathrm{diff}}\left(u_{i} u_{0}\right) .
$$

Since all $u_{i}$, for $i \geq 2$, are orthonormal to $u_{1}$ [i.e., $I_{\text {sum }}\left(u_{i} u_{1}\right)=0$ ], Eq. (5) demonstrates that these modes have a zero overlap integral with $u_{0}$ in a differential measurement. It can then be shown that only $u_{1}$, which has a nonzero overlap integral with $u_{0}$, has to be considered along with $u_{0}$ in the noise calculation $[4,16]$.

We note that the modes $u_{0}$ and $u_{1}$ have perfect interference visibility as shown by their complete overlap integral for the differential measurement, i.e., $I_{\text {diff }}\left(u_{0} u_{1}\right)=1$. In this regard, the measurement is analogous to a perfect homodyne measurement with a beam splitter. The two modes are equivalent to the two input beams of a beam splitter and the two halves of the multimode beam are equivalent to the two outputs. Therefore, similar to a homodyne measurement, the noise on the differential measurement is completely canceled when the flipped mode is occupied by a perfect squeezed vacuum, with the squeezed quadrature in phase with the coherent field of the $u_{0}(x)$ mode. Conversely, the same result is also obtained when the mode profiles of the squeezed and the coherent fields are interchanged. In order to avoid the effect of losses, we have chosen a squeezed vacuum mode $u_{0}(x)$. We would like to stress that this simplified explanation can be applied only because we have conveniently identified the two relevant transverse modes of the measurement. However, contrary to a homodyne measurement, the entire measurement is performed using a single beam. Furthermore, a more general analysis is not limited to only two-mode beams.

The experimental setup is shown in Fig. 3. A stable $\mathrm{Nd}$ :YAG $700 \mathrm{~mW}$ laser provides a $\mathrm{cw}$ single mode beam at $1064 \mathrm{~nm}$. A part of this beam is sent to a locked $\mathrm{MgO}: \mathrm{LiNbO}_{3}$ frequency doubling cavity. The $532 \mathrm{~nm}$ output of the frequency doubler is used to pump a degenerate optical parametric amplifier (OPA) that produces a stable $10 \mu \mathrm{W}$ squeezed beam in the $\mathrm{TEM}_{00}$ mode at $1064 \mathrm{~nm}$. The noise reduction of the OPA output is measured to be $3.5 \mathrm{~dB}$. Details of this squeezing system may be found in [17]. The flipped mode, $u_{1}(x)$, is produced by sending the remaining part of the initial $1064 \mathrm{~nm}$ laser beam through 


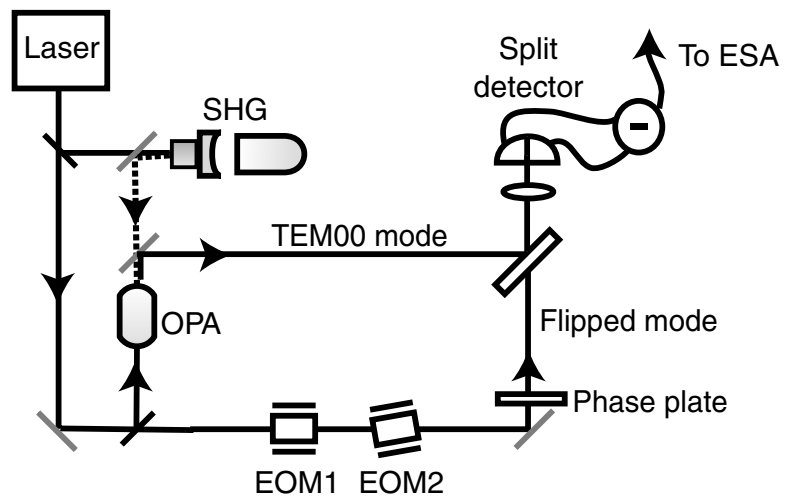

FIG. 3. Scheme of the experimental setup. SHG: second harmonic generator; OPA: optical parametric amplifier; EOM: electrooptic modulator; and ESA: electronic spectrum analyzer. The dashed lines correspond to light at $532 \mathrm{~nm}$, and the solid line to the light at $1064 \mathrm{~nm}$. The TEM00 mode is produced by the OPA and is a squeezed vacuum, the flipped mode is a coherent state.

a specially designed phase plate. This phase plate consists of two birefringent half-wave plates, one rotated by $90^{\circ}$ with respect to the other, forming the two halves $x<0$ and $x>0$ of the transverse plane. These elements introduce a phase shift of $180^{\circ}$ between the field amplitudes of the two halves. The squeezed output from the OPA is required to be superimposed onto the flipped mode with minimal loss. This is achieved by using a beam splitter that reflects $92 \%$ of the squeezed state and transmits $8 \%$ of the coherent state. The reflected output is then sent to a quadrant InGaAs detector (EPITAXX 505Q) with quantum efficiency greater than $90 \%$. Only two of the four quadrants, of dimensions $500 \mu \mathrm{m} \times 500 \mu \mathrm{m}$ each and with a dead zone between the pixels of $25 \mu \mathrm{m}$, are used in this experiment. A lens of focal length $30 \mathrm{~mm}$ is used to image the phase plate on the detector plane and to counteract the diffraction of the flipped mode, which undergoes an abrupt phase change and therefore contains high spatial frequency components.

Figure 4 shows the different noise levels monitored as a function of time when the relative phase between the coherent state and the squeezed state is chosen for maximum noise reduction. Because of the high stability of the various servoloops in the experimental setup, the actively locked operation of the setup can be kept for hours. The noise measured on the sum of the two halves (Fig. 4a), i.e., on the total beam, coincides with the shot noise level for the conditions of this experiment, as expected from the coherent beam, which is not affected by the presence of a squeezed vacuum in an orthogonal mode. The noise measured on each individual half (Fig. 4c) is reduced by $1.08 \pm 0.06 \mathrm{~dB}$ below the quantum noise limit. The fact that the intensity noise on each half of the beam is below the quantum noise limit, whereas the whole beam is at shot noise, shows the strong nonclassical characteristic of this multimode beam. This is corroborated by the experimental

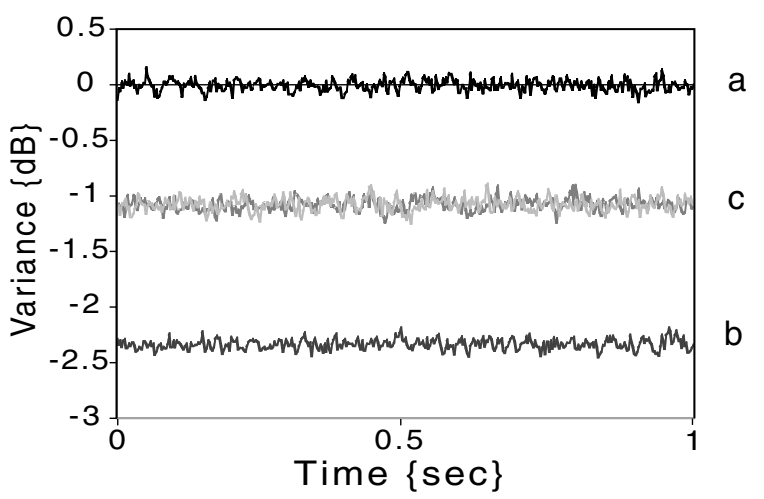

FIG. 4. Noise spectral density at $4.5 \mathrm{MHz}$ of the photocurrents as a function of time (resolution bandwidth $100 \mathrm{kHz}$ ). (a) Sum of the two photocurrents. (b) Difference between the photocurrents. (c) Noise on each detector.

data of Fig. 4b, which give the noise on the intensity difference between the two halves at $2.34 \pm 0.05 \mathrm{~dB}$ below the quantum limit. The results suggest that the beam is made of two strongly quantum correlated parts, indicating that a significant amount of spatial correlation has been created among the photons. With the measured noise reduction in the squeezed vacuum and a perfect setup (i.e., a perfect phase plate and a perfect mode matching between the two transverse modes), one would expect $2.5 \pm 0.2 \mathrm{~dB}$ of noise reduction on the difference between the two pixels. This demonstration is, to our knowledge, the first experiment in which spatial quantum effects have ever been observed in a bright beam of light.

This spatial noise correlation can now be used to improve the precision of displacement measurements in the image plane. For practical reasons, we have chosen to induce the displacement only in the coherent mode, before the mixing on the beam splitter. However this displacement is of the order of the nanometer, which is several orders of magnitude smaller that the relevant precision for the mode matching of the two transverse modes, and the theoretical prediction for the measurements is the same as if the displacement were done on the total beam. In order to produce a small controllable beam displacement in the frequency range of the previous measurements, we use two electro-optic modulators (EOMs) driven at $4.5 \mathrm{MHz}$. Figure 3 shows that EOM2 is slightly tilted with respect to the propagation of the light beam. When a voltage is applied across EOM2, a change in refractive index is induced and the transmitted beam experiences a parallel transverse displacement measured at about $3 \mathrm{~nm} / \mathrm{V}$. We introduce a modulation at $4.5 \mathrm{MHz}$ as signal for our displacement measurement which can be easily distinguished from the low frequency beam displacements induced by mechanical or acoustic vibrations. Apart from the parallel displacement, EOM2 will also introduce an unwanted phase modulation on the transmitted beam which is detrimental to our measurement. EOM1 of Fig. 3 is therefore used to compensate for this introduced phase modulation. When 


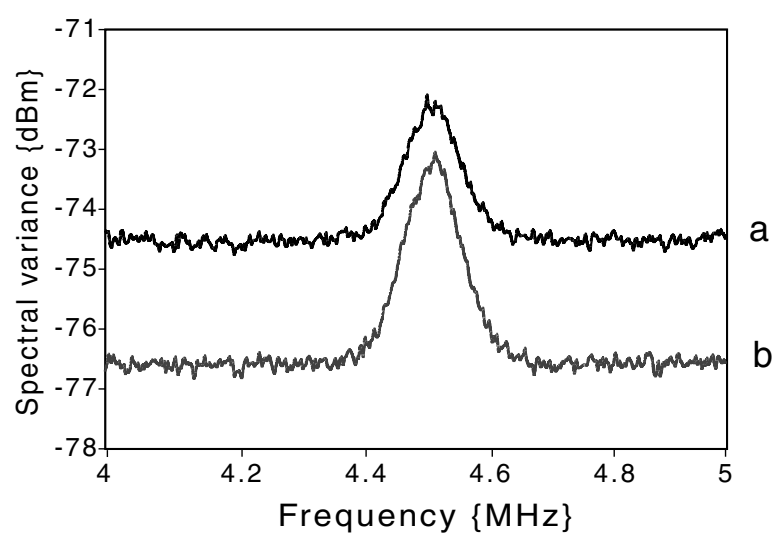

FIG. 5. Noise spectrum of the photocurrent difference in the presence of an oscillating displacement of amplitude $2.9 \AA$ and frequency $4.5 \mathrm{MHz}$ (resolution bandwidth: $100 \mathrm{kHz}$ ). (a) Using a coherent state of light. (b) Using the two-mode non classical state of light. This curve is obtained by averaging the signal over 10 successive traces.

correct gains are chosen for both modulators, the transmitted beam will not have any phase or amplitude modulation and is left only with pure transverse displacement modulation. Figure 5 shows the differential signal monitored by a spectrum analyzer when the light beam undergoes a displacement modulation with an amplitude of $2.9 \AA$. With a resolution bandwidth of $100 \mathrm{kHz}$, our setup recorded a modulation peak in the Fourier spectrum. Figure 5a shows the trace when vacuum instead of the squeezed vacuum is used in mode $u_{0}(x)$. Thus this noise floor gives the standard quantum limit in such a displacement measurement. The signal-to-noise ratio (SNR) of this measurement is 0.68 . When the two-mode nonclassical beam is utilized in the measurement (Fig. 5b), we obtain a SNR of 1.20. This gives an improvement of the displacement measurement sensitivity by a factor of 1.7 . The result is in agreement with the theoretical value calculated with the noise reduction reported in the previous paragraph. Similar measurements have been performed with a $10 \mathrm{kHz}$ resolution bandwidth (and therefore a longer measurement time) and the results show the same improvement of the SNR.

Our results demonstrate that multimode nonclassical states of light can be utilized to improve the optical measurement of small displacements. The noise floor of displacement measurements can actually be reduced to below the standard quantum limit. Of particular relevance are the potential usage of multimode squeezed light in atomic force microscopy and biological microscopy. Though our experimental demonstration is restricted to one-dimensional displacement measurements, it can be extended to two-dimensional displacement measurements with more complex forms of multimodal nonclassical light.

We would like to thank L. Lugiato and M. Kolobov for many enlightening discussions and CSIRO, Sydney, for the manufacturing of the special phase plate. This work is funded by the European Project No. IST-2000-26019 "Quantum images," the Centre National de la Recherche Scientifique, and the Australian Research Council.

[1] H-A Bachor, A Guide to Experiments in Quantum Optics (Wiley-VCH, Weinheim, 1998).

[2] M. Xiao, L. A. Wu, and H. J. Kimble, Phys. Rev. Lett. 59, 278 (1987); P. Grangier et al., Phys. Rev. Lett. 59, 19 (1987).

[3] E. Polzik, J. Carri, and H. J. Kimble, Appl. Phys. B 55, 279 (1992); F. Marin, A. Bramati, V. Jost, and E. Giacobino, Opt. Commun. 140, 146 (1997).

[4] C. Fabre, J. B. Fouet, and A. Maître, Opt. Lett. 25, 76-78 (1999).

[5] Seng-Tiong Ho, P. Kumar, and J. H. Shapiro, Phys. Rev. A 37, 2017 (1988).

[6] M. Kolobov and C. Fabre, Phys. Rev. Lett. 85, 3789 (2000).

[7] J. P. Poizat, T. Chang, and P. Grangier, Phys. Rev. A 61, 043807 (2000); J. P. Hermier et al., IEEE J. Quantum Electron. 37, 87 (2001).

[8] L. A. Lugiato, A. Gatti, and H. Wiedemann, in Quantum Fluctuations, Proceedings of the Les Houches Summer School (North-Holland, Amsterdam, 1997), p. 431.

[9] M. I. Kolobov, Rev. Mod. Phys. 71, 1539 (1999).

[10] A. Joobeur et al., Phys. Rev. A 53, 4360 (1996).

[11] L. A. Lugiato and I. Marzoli, Phys. Rev. A 52, 4886 (1995); I. Marzoli, A. Gatti, and L. A. Lugiato, Phys. Rev. Lett. 78, 2092 (1997).

[12] C. A. J. Putman et al., J. Appl. Phys. 72, 6 (1992).

[13] C. Boccara, D. Fournier, and J. Badoz, Appl. Phys. Lett. 36, 130 (1980).

[14] H. Kojima et al., Biophys. J. 73, 2012 (1997).

[15] D. P. E. Smith, Rev. Sci. Instrum. 66, 3191 (1995).

[16] V. Delaubert et al. [J. Opt. A (to be published)].

[17] B. C. Buchler et al., Phys. Rev. A 65, 011803(R) (2002). 